\section{Membrane potential dynamics of axons in cultured hippocampal neurons probed by second-harmonic-generation imaging}

\author{
Mutsuo Nuriya and Masato Yasui \\ Keio University School of Medicine, Department of \\ Pharmacology, 35 Shinanomachi, Shinjuku, \\ Tokyo 160-8582, Japan
}

\begin{abstract}
The electrical properties of axons critically influence the nature of communication between neurons. However, due to their small size, direct measurement of membrane potential dynamics in intact and complex mammalian axons has been a challenge. Furthermore, quantitative optical measurements of axonal membrane potential dynamics have not been available. To characterize the basic principles of somatic voltage signal propagation in intact axonal arbors, second-harmonic-generation (SHG) imaging is applied to cultured mouse hippocampal neurons. When FM4-64 is applied extracellularly to dissociated neurons, whole axonal arbors are visualized by SHG imaging. Upon action potential generation by somatic current injection, nonattenuating action potentials are recorded in intact axonal arbors. Interestingly, however, both current- and voltage-clamp recordings suggest that nonregenerative subthreshold somatic voltage changes at the soma are poorly conveyed to these axonal sites. These results reveal the nature of membrane potential dynamics of cultured hippocampal neurons, and further show the possibility of SHG imaging in physiological investigations of axons. () 2010 Society of Photo-Optical Instrumentation Engineers. [DOI: 10.1117/1.3365135]
\end{abstract}

Keywords: second harmonic generation; imaging; cells; action potential; axon; voltage; neuron; culture.

Paper 10010LR received Jan. 10, 2010; revised manuscript received Feb. 16, 2010; accepted for publication Feb. 22, 2010; published online Mar. 23, 2010.

\section{Introduction}

Axons extend elaborate arbors over a long distance to form synaptic contacts that underlie communication between neurons. In addition to their role as a reliable cable that faithfully propagates action potentials to presynaptic terminals, a more complex information processing capacity of axons has recently been proposed. ${ }^{1,2}$ However, due to the thin and elaborate structures of mammalian axons, most investigations on their electrical properties have been restricted to indirect measures such as calcium imaging and theoretical calculations. Second-harmonic-generation (SHG) imaging enables quanti-

Address all correspondence to: Mutsuo Nuriya. Tel: 81-3-5363-3750; Fax: $81-$ 3-3359-8889; E-mail: mnuriya@sc.itc.keio.ac.jp tative measurements of membrane potential dynamics in neuronal structures that are hardly accessible by conventional electrophysiological techniques. ${ }^{3,4}$ In slice preparations, however, visualization and following recordings of SHG signals at distal neurites of the patch-clamped neuron are limited by the slow diffusion of the SHG dye from the injection site of the soma. In particular, axonal structures are hardly visible in these systems. In this study, we employed low density dissociated cultured hippocampal neurons to visualize axonal arbors by SHG imaging, and utilized them to quantitatively measure membrane potential dynamics in axons.

\section{Methods}

Hippocampal neurons obtained from E17 mice were plated onto poly-L-lysine coated coverslips (Iwaki, Tokyo, Japan) at a density of $1 \times 10^{5}$ cells $/ 60 \mathrm{~mm}$ dish and maintained in Neurobasal medium supplemented with 2\% B27, 1\% GlutaMAX, and $1-\mu \mathrm{g} / \mathrm{ml}$ gentamicin (Invitrogen, Carlsbad, California) until use (10 to 20 days in vitro).

Cultured hippocampal neurons on coverslips were transferred to the imaging buffer $(125-\mathrm{mM} \mathrm{NaCl}, 5-\mathrm{mM} \mathrm{KCl}$, 10-mM dextrose, 1-mM $\mathrm{MgCl}_{2}, 2-\mathrm{mM} \mathrm{CaCl}_{2}, 10-\mathrm{mM}$ HEPES, pH 7.3 with $2 \%$ B27 supplement) containing $20-\mu \mathrm{M}$ FM4-64 (SynaptoRed C2, Biotium, Hayward, California) and intracellularly loaded with 200- $\mu \mathrm{M}$ Alexa Fluor 488 hydrazide (Invitrogen) through a patch pipette. Neurons were placed under the FV1000MPE system (Olympus, Tokyo, Japan) equipped with a MaitaiHP femtosecond laser (Newport, Mountain View, California). SHG signals generated from 1000-nm laser illumination were collected at the bottom detector after a 500/20-nm bandpass filter (Asahi Spectra, Tokyo, Japan). Fast recordings of SHG signals were performed by point-scan protocol and acquired the SHG signal directly from the photomultiplier tube (PMT) to the data acquisition board (National Instruments, Austin, Texas) after a preamplifier (NF Corporation, Kanagawa, Japan). "Multipoint scan" software (Olympus) was used for point-scan recordings, where 30 to $40 \mathrm{msec}$ of laser illumination was applied to the same target point 100 times with 500 -msec intervals. During these 100 pulses, neurons were stimulated every other time, $10 \mathrm{msec}$ after the onset of the laser illumination.

Electrophysiological recordings were performed using MultiClamp 700B (Molecular Devices, Sunnyvale, California). A glass pipette (6 to $12 \mathrm{M} \Omega$, Warner Instruments, Hamden, Connecticut) was filled with internal solution containing 10-mM NaCl, 10-mM KCl, 135-mM KMeSO, $2.5-\mathrm{mM}$ MgATP, 0.3-mM NaGTP, 10-mM HEPES, and pH 7.3. For voltage-clamp experiments, a cesium gluconate-based internal solution (110-mM CsGluconate, 20-mM CsCl, 2-mM EGTA, 10-mM HEPES, 0.3-mM NaGTP, 2-mM MgATP, and 5-mM QX-314) was used together with 1-mM $\mathrm{NiCl}_{2}$ applied to the bath. All recordings were performed at room temperature.

Data were analyzed by custom-written software (MATLAB, Mathworks, Natick, Massachusetts) and statistical significance was tested using a two-tailed unpaired t-test. All data are shown as the mean \pm standard error of the mean, except for those of "minimum estimated distance," where the

1083-3668/2010/15(2)/020503/3/\$25.00 @ 2010 SPIE 
mean \pm standard deviation was used to describe variations in the recording points. SHG signals of control and stimulation groups were averaged and then normalized using signals preceding the stimulation, and the percent change in SHG was calculated by dividing the differences between groups by the value of the control group at each time point. To check SHG responses on current injection, all data were aligned to the peaks of voltage deflections recorded electrophysiologically. In voltage-clamp experiments, SHG responses to voltage steps were calculated from data collected between 15 to $25 \mathrm{msec}$ after the onset of voltage steps.

\section{Results and Discussion}

To visualize the entire structures of neurons, including axons and dendrites, we extracellularly applied the SHG dye FM 4-64 to low-density dissociated cultured mouse hippocampal neurons. These neurons have little overlap between each other and therefore, when FM 4-64 is applied in the bath, the entire neuronal structures including thin and elaborate axonal arbors should be readily decorated by FM 4-64 and visible by SHG imaging. Indeed, the entire neuronal structures are strongly stained by FM 4-64, as revealed by two-photon fluorescence, and can be visualized by SHG imaging [Fig. 1(a)]. Therefore, we employed this system to record the membrane potential dynamics of intact axons in a quantitative manner by using SHG imaging. To unambiguously trace axons originating from the patch-clamped neuron, freely diffusible Alexa 488 dye was loaded into neurons intracellularly through the patch pipette. Since there was no detectable two-photon fluorescence signal from Alexa 488 with the 1000-nm laser illumination used for SHG imaging, a single-photon laser at $488 \mathrm{~nm}$ was used to visualize the Alexa 488 signals. When visualized by Alexa 488 fluorescence, axonal processes were easily identifiable based on their thin and untapered structures that show extensively curved morphologies reaching far from the soma [Fig. 1(a)]. To confirm this point, an immunocytochemical analysis was performed. Indeed, as reported previously, ${ }^{5}$ Tau-1 staining revealed that axonal structures have very distinct morphologies from those of dendrites stained by MAP2, and could be readily distinguished [Fig. $1(\mathrm{~b})]$. Therefore, as described previously, ${ }^{6}$ these morphological parameters were utilized to identify axons. Finally, to characterize the voltage sensitivity of SHG signals in this system, neurons were voltage-clamped in the presence of voltage-gated ion channel blockers (see Sec. 2) and the SHG responses at various voltage steps were recorded [Fig. 1(c)]. As reported previously, ${ }^{3}$ SHG signals changed with membrane potential in a linear manner at $-10 \%$ SHG change/+100 $\mathrm{mV}$ voltage change within and even beyond the physiological voltage fluctuation range, confirming the ability of SHG imaging to quantitatively measure membrane potential changes in this system. For better comparison between the SHG signal changes and membrane potential dynamics, the sign of SHG changes were reversed in the rest of this study.

Having established the SHG recordings in cultured hippocampal neurons, we then investigated the propagation of voltage signals from the soma to axonal arbors. First, the SHG response to an action potential was examined. The action potential was initiated by injecting depolarizing current at the soma for $10 \mathrm{msec}$. When SHG signals were recorded in the middle of axon collaterals, they clearly showed changes in the
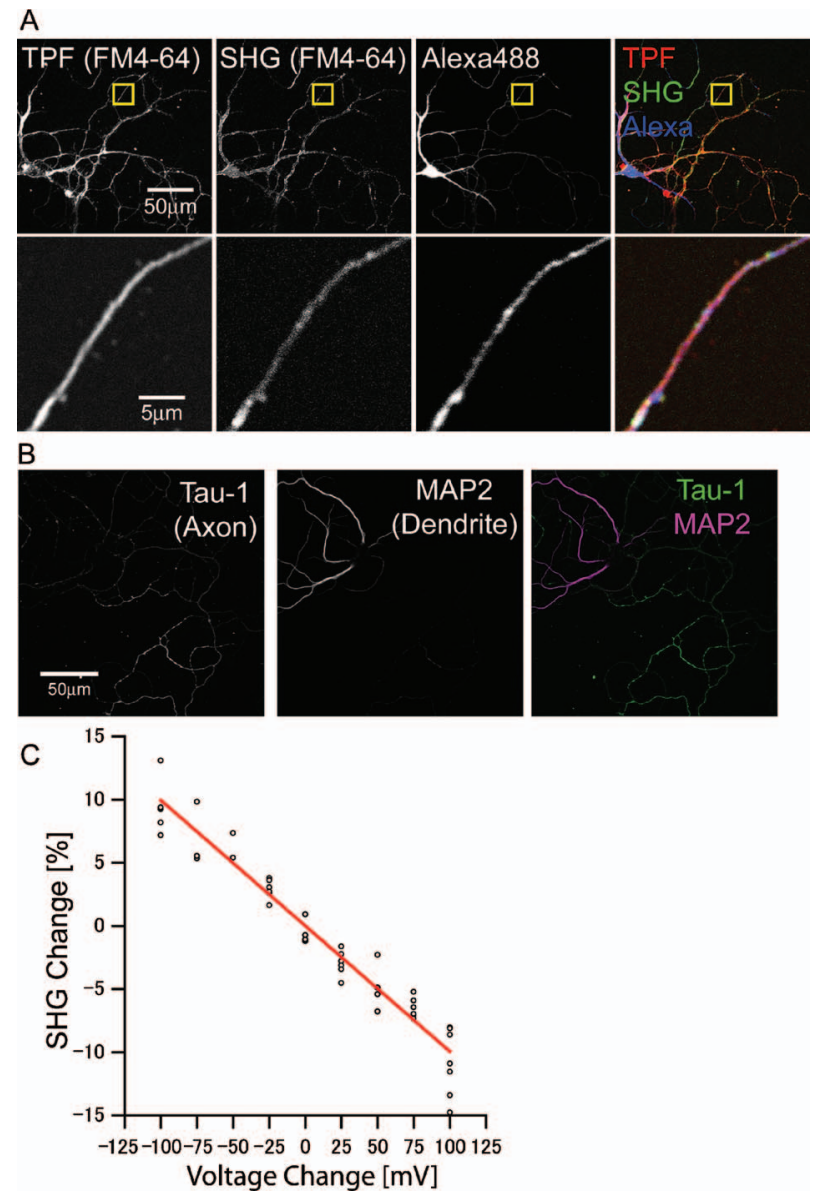

Fig. 1 Characterization of SHG signals from cultured neurons. (a) Visualization of SHG signals from cultured hippocampal neurons. Twophoton fluorescence (TPF) and SHG signals from bath-applied FM4-64 were visualized simultaneously. Single-photon fluorescence of intracellularly applied Alexa 488 was collected separately and merged together. Lower panels show a higher magnification view of the selected region in the top panels. (b) Immunocytochemical analysis of the hippocampal neurons. Axons and dendrites were stained with an anti-Tau-1 and anti-MAP2 antibody, respectively. (c) Voltage sensitivity of SHG signals. Neurons were voltage-clamped, and the membrane potential was changed systematically from -100 to $+100 \mathrm{mV}$, starting from a resting membrane potential of $-65 \mathrm{mV}$ (i.e., -165 to $+35 \mathrm{mV}$ ). SHG changes at the soma were calculated and plotted against the membrane potential changes. The red line shows the linear fit of the data $\left(y=-0.099 x, R^{2}=0.94\right.$, and $n=46$ from 11 neurons).

amplitude corresponding to the shape of the action potential that was electrophysiologically recorded at the soma [Fig. 2(a)]. The minimum estimated distance from the soma to the SHG recording sites was $205.9 \pm 57.9 \mu \mathrm{m}(n=5)$. When the peak amplitudes of the SHG responses were compared to those recorded at the soma and at axons, there was no significant difference between these two groups $(p=0.54, n=5$, t-test). This indicates that in these cultured hippocampal neurons, action potential propagates through axon branches in full magnitude over a long distance.

Propagation of an action potential into axonal arbors can be supported by an active regenerative process, or it can be mediated passively through the cable. To investigate the extent of this passive propagation, the same experiment was 

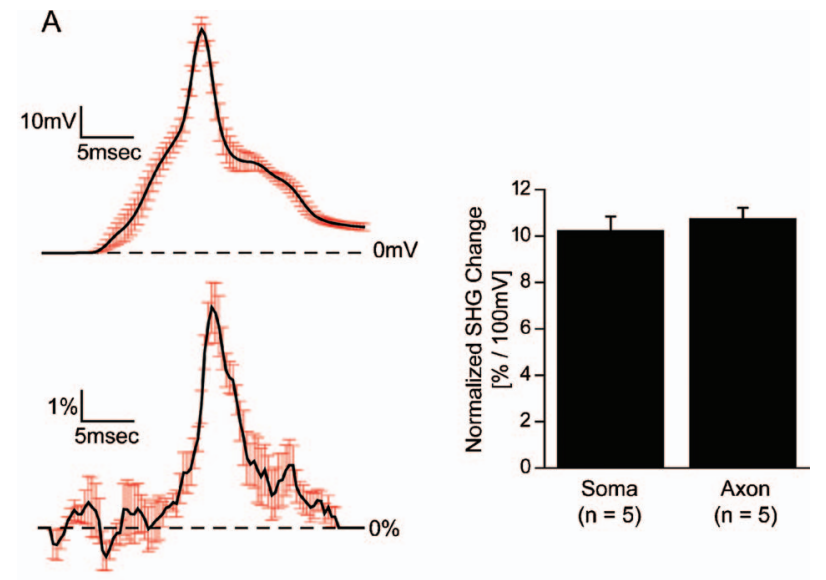

B
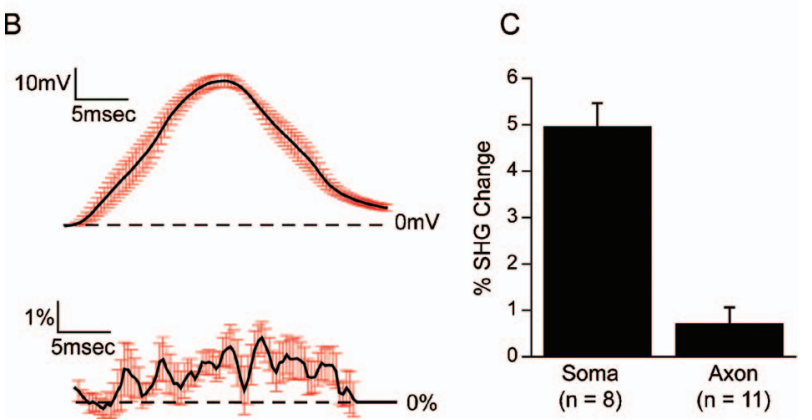

Fig. 2 Propagation of somatic voltage changes in axons. (a) Action potential-induced SHG changes in axons. The upper left panel shows the voltage changes recorded at the soma, and the lower left panel shows SHG changes recorded in axons. Data are shown as the mean (black) \pm SEM (red) from five neurons. Note that the sign of SHG change is reversed for the purpose of visualization. The right panel shows a comparison of the peak amplitude of SHG signal changes normalized to the action potential with a $100-\mathrm{mV}$ amplitude. (b) SHG changes at axons in the presence of TTX. Neurons were held under current-clamp conditions in the presence of $1-\mu \mathrm{M}$ TTX and injected with a depolarizing current pulse. The voltage change at the soma (upper panel) and corresponding changes of SHG recorded at axons (lower panel) are shown. Data shown is the mean (black) \pm SEM (red) from 12 recordings from seven neurons. (c) Neurons were held under voltage-clamp configuration and $50-\mathrm{mV}$ voltage pulses were applied to the soma. SHG responses at the soma ( $n=8$ from six neurons) and axons ( $n=11$ from six neurons) were measured. Data in the panel are presented is the mean \pm SEM.

performed in the presence of $1-\mu \mathrm{M}$ tetrodotoxin (TTX), a specific blocker of voltage-gated sodium channels that trigger the regenerative action potential. As expected, the action potential was completely abrogated under this condition [Fig. 2(b), top panel]. Surprisingly, the SHG responses at axons were greatly attenuated; this attenuation was much more pronounced than the corresponding reduction in somatic voltage changes, barely reaching above the noise level during the nearly $50-\mathrm{mV}$ changes at the soma [Fig. 2(b), bottom panel]. To obtain a better estimation of passive propagation of the somatic voltage changes, neurons were held under the voltage-clamp mode in the presence of voltage-gated ion channel blockers. Upon 50-mV depolarization, the SHG at the soma showed a $5 \%$ signal change $(4.94 \pm 0.52 \%, n=8)$, consistent with the previous results [Fig. 2(c)]. Interestingly, however, the SHG changes at axons were significantly smaller than those at the soma $(p<0.0001$, t-test), reaching less than $1 \%$ in amplitude for the same $50-\mathrm{mV}$ voltage change at the soma $(0.70 \pm 0.36 \%, n=11)$ [Fig. 2(c)]. The minimum estimated distances from the soma for TTX and voltage-clamp experiments were $120.3 \pm 50.2 \mu \mathrm{m} \quad(n=12) \quad$ and $118.9 \pm 54.0 \mu \mathrm{m} \quad(n=11)$, respectively. Due to very small SHG signal changes in axons and uncertainty of the actual length of the target sites from the soma, the length constant of the axons in this system could not be estimated from our measurements. Nevertheless, these results suggest that propagation of nonregenerative voltage signals from the soma to axons is very inefficient in these cultured neurons. These results appear to be in contrast to data obtained from patchclamp recordings of axonal bleb or mossy fiber terminals, ${ }^{7,8}$ both of which suggested the effective transduction of subthreshold voltage signals from the soma to axon terminals. A previous electron microscopy study using a similar culture system estimated the diameter of axons below $0.3 \mu \mathrm{m} .{ }^{9}$ In addition, it was evident that the recorded axons extended extremely elaborate collaterals [Figs. 1(a) and 1(b)], which together with the influence of diameter would critically affect the cable properties of axons. Therefore, while we cannot exclude the possibility that the discrepancy between our study and patch-clamp studies arise from differences between the systems and nature of neurons being used, it is intriguing to hypothesize that it also reflects the differences in the electrical properties of axons due to differences in their morphologies. ${ }^{1}$

\section{Acknowledgments}

We thank Olympus Corporation for their collaborative efforts, Manae Imamura for excellent technical assistance. This work was supported by the Ministry of Education, Culture, Sports, Science, and Technology of Japan, as well as the Kanae Foundation and the Uehara Foundation.

\section{References}

1. I. Segev and E. Schneidman, "Axons as computing devices: basic insights gained from models," J. Physiol. Paris 93(4), 263-270 (1999).

2. D. Debanne, "Information processing in the axon," Nat. Rev. Neurosci. 5(4), 304-316 (2004).

3. M. Nuriya, J. Jiang, B. Nemet, K. B. Eisenthal, and R. Yuste, "Imaging membrane potential in dendritic spines," Proc. Natl. Acad. Sci. U.S.A. 103(3), 786-790 (2006).

4. L. Sacconi, D. A. Dombeck, and W. W. Webb, "Overcoming photodamage in second-harmonic generation microscopy: real-time optical recording of neuronal action potentials," Proc. Natl. Acad. Sci. U.S.A. 103(9), 3124-3129 (2006)

5. S. Kaech and G. Banker, "Culturing hippocampal neurons," Nat. Protoc. 1(5), 2406-2415 (2006).

6. P. J. Mackenzie, M. Umemiya, and T. H. Murphy, "Ca2+ imaging of CNS axons in culture indicates reliable coupling between single action potentials and distal functional release sites," Neuron 16(4), 783 795 (1996).

7. Y. Shu, A. Hasenstaub, A. Duque, Y. Yu, and D. A. McCormick, "Modulation of intracortical synaptic potentials by presynaptic somatic membrane potential," Nature (London) 441(7094), 761-765 (2006).

8. H. Alle and J. R. Geiger, "Combined analog and action potential coding in hippocampal mossy fibers," Science 311(5765), 1290-1293 (2006).

9. W. P. Bartlett and G. A. Banker, "An electron microscopic study of the development of axons and dendrites by hippocampal neurons in culture. II. Synaptic relationships," J. Neurosci. 4(8), 1954-1965 (1984). 